in its contracted condition, or in a small centrally situated nebula, the vision is often improved by a small optical iridectomy, and in some instances this may be combined with the division of an adhesion of the iris to the posterior surface of the cornea. I do not as a rule advise the simple division of anterior synechiae, as from my own experience I do not think that they often give rise to much trouble. For staphylomatous cornea and buphthalmic eyes which are protruding between the lid or which are very unsightly, a Mule's operation is advisable, using a small glass ball, and stitching the sclera together with a purse-string suture of silk, a separate stitch being made for the conjunctiva. If this method is followed, extrusion of the ball is extremely rare.

\section{Other Complications}

Gonorrhoeal arthritis, although not uncommon in the mothers, is rare in the babies. We have had only one such case in $35^{\circ}$ children. This child had both ankles and knee-joints severely affected and subsequently died of heart failure.

Rhinitis is a frequent accompaniment of ophthalmia neonatorum. The gonococcus can be found in the nose when there is a large quantity of discharge, or when there is practically none. If there is much discharge there are always immense quantities of other organisms, and I think that the rhinitis is attributable far more to these organisms than to the gonococcus.

Purulent mucocele is often mistaken for ophthalmia neonatorum. In sume of these cases the gonococcus has been found in the discharge, although the pneumococcus is more frequent.

\section{SOME OBSERVATIONS ON THE CONTROL OF MEASLES EPIDEMICS}

\author{
r.Y
}

PERCY STOCKS, M.D., D.P.H.

READER IN MEDICAL STATISTICS, UNIVERSITY OF LONDON

The opinion is often expressed that measles epidemics cannot be controlled. The basis for this view seems to be that isolation is generally useless because the harm is usually done before the diagnosis is made, and that school closure is neither practicable nor effective in suppressing an epidemic. Dr. Forbes, medical officer of health for Brighton, has recently suggested that the exclusion of home contacts attending infected schools might also be abandoned. In view of advances which have been made in our knowledge of the epidemiology of measles it seems worth while to ask ourselves how far epidemics could be modified, if it were so desired, by applying the knowledge we now have.

In an article ${ }^{1}$ entitled "The mechanism of a measles epidemic," published in April of last year, I expressed the view that "the only hope of really preventing these epidemics altogether is through the immunity factor," and that "we must hope for either a simple method of inoculation which will produce a more or less permanent immunity, or else a simple method of rendering an attack abortive and harmless, such as the proposed method of inoculating contacts with their parent's blood, whilst at the same time allowing it to produce a permanent immunity." Considerable research has been done recently on the experimental production of passive immunity in children by the use of human convalescent serum, and this was well summarized in the recent Report of the Medical Officer of Health and School Medical Officer on the Measles Epidemic of 1929-30 (London County Council, 1931), and amplified by Dr. D. N. Nabarro and Dr. A. G. Signy in their paper in the Journal. ${ }^{2}$ In their series of 625 children treated with human serum before the fifth day after exposure to measles infection, only twelve developed measles, as compared with an expectation of several hundreds, and all the cases were mild in form. The passive immunity produced in this way lasts, we are told, two to four weeks. If the serum is injected on the sixth to the ninth day after exposure to infection partial protection is afforded, an attenuated attack resulting, which produces permanent active immunity.

\section{Sero-protection in Relation to Epidemics}

Provided that the serum is available, we now have a means, therefore, of protecting all home contacts to measles cases for upwards of a month, or, better still, of rendering them permanently immune by allowing them to have an attenuated attack. Moreover, we could, if so desired, suppress an epidemic in any school by treating all unprotected children with convalescent serum, but in this case scarcely any of the treated children would receive anything but a temporary passive immunity, and since an epidemic lasts several months the school would probably be again invaded by measles when this immunity had waned and before the infectiousness had declined to the normal level. There are, of course, practical difficulties in the way of any such operations on a large scale-in particular, the difficulty of obtaining sufficient serum and of overcoming prejudice against such a procedure-but it is by no means unlikely that these difficulties may be removed. It is not, therefore, merely a matter of academic interest to estimate what effect. could be produced on a measles epidemic by giving certain groups of children a passive immunity at a certain phase of the epidemic.

The first question to ask is: What groups of children should be immunized in order to produce the maximal salutary effect? Some light is thrown upon this by the figures in Table I, which were obtained from an analysis of the card records of measles notifications in the metropolitan borough of Battersea during 1925-28. (This was

\begin{tabular}{|c|c|c|c|c|c|c|c|c|c|c|c|}
\hline & & \multicolumn{9}{|c|}{ Age of "Secondary Cace" } & \multirow{2}{*}{ Total } \\
\hline & & $0-1$ & $1-2$ & $2-3$ & $3-4$ & $4-5$ & $5-5$ & $6-7$ & $7-8$ & $8-9$ & \\
\hline \multirow{9}{*}{ 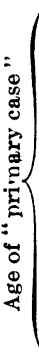 } & $0-1$ & - & - & 2 & 3 & 1 & 2 & - & - & - & 8 \\
\hline & $1-2$ & - & 2 & 3 & 3 & 3 & 1 & - & - & - & 12 \\
\hline & $2-3$ & 8 & 9 & - & 3 & 6 & 3 & 1 & - & - & 30 \\
\hline & $3-4$ & 9 & 17 & 14 & 1 & 4 & 6 & 3 & 1 & - & 55 \\
\hline & $4-5$ & 3 & 26 & 30 & 12 & 2 & 5 & 4 & 3 & 5 & 90 \\
\hline & $5-6$ & 20 & 37 & 38 & 34 & $<8$ & 5 & 7 & 3 & 4 & 176 \\
\hline & $6-7$ & 5 & 15 & 9 & $\therefore 1$ & 16 & 8 & 2 & 1 & 5 & 82 \\
\hline & $7-8$ & 2 & 4 & - & 5 & 1 & 1 & 2 & - & 3 & 18 \\
\hline & $8-9$ & 1 & 1 & 2 & 4 & - & 3 & 2 & - & 2 & 15 \\
\hline \multicolumn{2}{|c|}{ To'al } & 48 & III & 98 & 86 & 61 & 34 & $\approx 1$ & 8 & 19 & 486 \\
\hline
\end{tabular}

made possible by the courtesy of the medical officer of health, Dr. G. Quin Lennane.) The table shows the ages of 486 children under 9 years of age who contracted measles from another child in the same house, developing the rash ten to thirteen days later than the first child. These I have termed "'secondary cases," and the age of the child from whom the disease was contracted, termed the " primary case," is also shown in each instance. - It is readily seen from the table that the seendary cases are, on the whole, much younger than the primary cases; actually the mean age of the primaries is 5.3 years, and 60 per cent. are aged 5 or over, compared with 3.2 years and 17 per cent. for the secondaries. 
In Fig. 1 the contrast is shown more clearly, and it is evident that the chief culprits in taking home infection to their brothers and sisters are the 5-year-olds. At the foot of the diagram is shown the age distribution of the 176 children who contracted measles at home from these

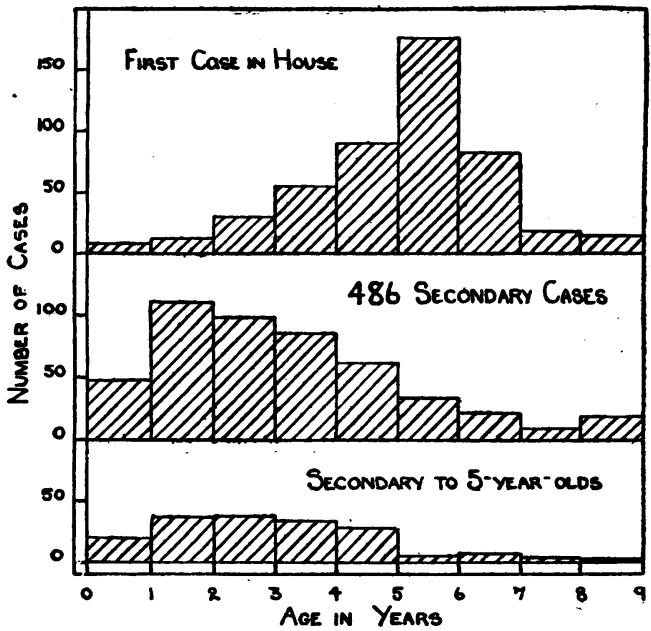

Fig. 1.-Age distribution of primary and secondary measles cases in households in Battersea, 1925-28.

5 -year-olds ; 137 of them, or more than three-quarters, were of ages $1,2,3$, or 4 years. In the present paper I shall only attempt to estimate the effect of producing temporary passive immunity by means of convalescent serum or parent's serum, in two groups of children who have never had measles-(1) all home contacts to measles cases, (2) all 5-year-old children-these being the most obvious groups to deal with in any attempt to modify an epidemic.

Effect of Immunizing Home Contacts

In Fig. 2 is shown the mean curve of measles incidence in Battersea, obtained by tabulating the numbers of notified cases in successive periods of ten days in each

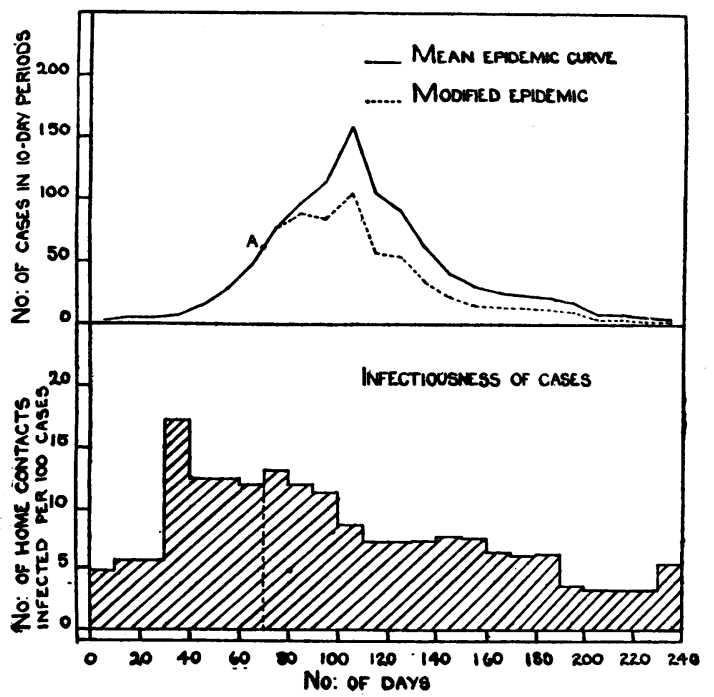

FIG. 2.-Measles epidemics in Battersea, 1926, 1928. Mean trend of incidence and infectiousness in nine districts.

of the nine wards of the borough and in two epidemics of 1926 and 1928 . The eighteen small epidemics, averaging about 500 cases in each, were then made synchronous by bringing all the " peaks" of the curves, or periods of ten days having the maximum incidence, together, and then summing the cases in corresponding periods as measured forwards and backwards from the peak period. As I have shown from St. Pancras data, ${ }^{3}$ measles epidemics move across the map, each district producing its epidemic curve of similar form and duration, but starting at different times as determined by a sudden upset in the balance between infectiousness and immunity factors; hence to obtain a typical curve it is necessary to proceed in this way. The curve in Fig. 2 is compiled from 8,649 cases, and the frequencies reduced to a total of 1,000 cases in 240 days-(namely, the peak period of ten days, ten periods preceding and thirteen periods succeeding it). In the lower portion is shown the ratio of the number of secondary cases which follow ten to thirteen days later in the same houses to the total incidence of cases, this being a measure of the " apparent infectiousness " 4 of the disease at the time of infection. This shows that a sudden rise in apparent infectiousness takes place at the very start of the epidemic, and it then gradually falls to a low value by the end of the epidemic. The details of total incidence and secondary cases are given in the first few columns of Table II, and in the fifth column is shown the incidence reduced to a total of 1,000 .

Supposing that in a district where a meas'es epidemic of 1,000 cases is to be expected from previous experience,

\begin{tabular}{|c|c|c|c|c|c|c|c|c|c|}
\hline \multirow{3}{*}{\multicolumn{2}{|c|}{$\begin{array}{c}\text { Periods } \\
\text { of Ten } \\
\text { Days }\end{array}$}} & \multirow{3}{*}{$\begin{array}{c}\text { Total } \\
\text { Inci- } \\
\text { dence }\end{array}$} & \multirow{3}{*}{$\begin{array}{c}\text { No. of } \\
\text { Second- } \\
\text { ary Cases } \\
\text { to thes? }\end{array}$} & \multirow{3}{*}{$\begin{array}{l}\text { Ratio } \\
\text { per } \\
\text { cent. }\end{array}$} & \multirow{3}{*}{$\begin{array}{c}\text { Inci- } \\
\text { dence in } \\
\text { Epi- } \\
\text { demic } \\
\text { of } 1,000\end{array}$} & \multirow{3}{*}{$\begin{array}{c}\text { No. } \\
\text { Aged } \\
5\end{array}$} & \multicolumn{3}{|c|}{$\begin{array}{l}\text { Expected Result of Im- } \\
\text { muniz:ng all 5-year olds }\end{array}$} \\
\hline & & & & & & & \multicolumn{2}{|c|}{ No. Suppressed } & \multirow{2}{*}{$\begin{array}{l}\text { Result- } \\
\text { ing Inci- } \\
\text { den:e }\end{array}$} \\
\hline & & & & & & & Aged 5 & O.hers & \\
\hline \multirow{10}{*}{ 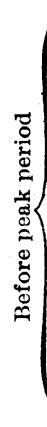 } & 10 & 19 & - & - & 2 & - & - & - & 2 \\
\hline & 9 & 45 & $4)$ & \multirow{2}{*}{5.6} & 5 & 1 & - & - & 5 \\
\hline & 8 & 44 & & & 5 & 1 & - & - & 5 \\
\hline & 7 & 58 & 10 & 17.3 & 7 & 2 & - & - & 7 \\
\hline & 6 & 142 & $31)$ & \multirow{2}{*}{12.5} & 16 & 4 & - & - & 16 \\
\hline & 5 & 249 & 18 & & 29 & 6 & - & - & 29 \\
\hline & 4 & 410 & 49 & 12.0 & 48 & 11 & - & - & 48 \\
\hline & 3 & 663 & 87 & 13.1 & 77 & 17 & - & - & 77 \\
\hline & 2 & 828 & 99 & 12.0 & 93 & 21 & 7 & - & 83 \\
\hline & 1 & S77 & 111 & 11.4 & 113 & 25 & 25 & 5 & 83 \\
\hline \multicolumn{2}{|c|}{ Maximun } & 1,337 & 119 & 8.7 & 158 & 35 & 33 & 22 & 103 \\
\hline \multirow{13}{*}{ 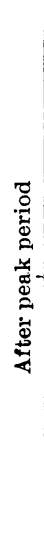 } & 1 & 500 & 79 & \multirow{2}{*}{7.2} & 104 & 23 & 12 & 35 & 57 \\
\hline & 2 & 777 & $42 J$ & & $\subseteq 0$ & 20 & 1 & 36 & 53 \\
\hline & 3 & 520 & 38 & 7.3 & CO & 13 & - & 26 & 34 \\
\hline & 4 & 349 & $\varepsilon 7$ & 7.7 & 40 & 9 & - & 18 & 22 \\
\hline & 5 & 262 & 20 & 7.6 & 30 & 7 & - & 14 & 16 \\
\hline & 6 & 223 & 14 & 6.3 & 26 & 6 & - & 12 & 14 \\
\hline & 7 & 202 & 12 & 6.0 & 24 & 5 & - & 11 & 13 \\
\hline & 8 & 196 & 12 & 6.1 & 22 & 5 & - & 10 & 12 \\
\hline & 9 & 166 & 6 & 3.6 & 19 & 4 & - & 9 & 10 \\
\hline & 10 & 78 & & \multirow{4}{*}{3.3} & 9 & 2 & - & 5 & 4 \\
\hline & 11 & 76 & 4 & & 9 & 2 & - & 5 & 4 \\
\hline & 12 & 55 & 2 & & 6 & 1 & - & 4 & 2 \\
\hline & 13 & 43 & - & & 5 & 1 & - & 3 & 2 \\
\hline \multicolumn{2}{|c|}{ Totals... } & 8,649 & 786 & 9.1 & 1,000 & 221 & 78 & 215 & 707 \\
\hline
\end{tabular}

as soon as the incidence. has risen to $\mathbf{5 0}$ cases in ten days, that is at point A, about the seventieth day on the time scale in Fig. 2, every home contact who has never had measles is immunized within five days from appearance of the rash in the primary case, and that this is continued until the epidemic is over; what would be the expected effect? Every secondary case below the dotted line in Table II would be suppressed, a total of 673 cases in a 
total epidemic of 8,649 , or 78 cases in a total epidemic of 1,000 . The "secondary" cases as here defined would be accompanied by about half as many others with incubation periods of fourteen to eighteen days ${ }^{6}$ who would also escape, making 117 in all. Owing to the practice of keeping contacts at home during the infective period it may be assumed that they would not have infected other children outside their own houses, and hence the total effect would be limited to reducing the epidemic from 1,000 to 883 . A few of the 117 children might contract measles from outside sources before the epidemic was over, owing to the rapid loss of their temporary passive immunity, but the vast majority of them would escape for another two years (in London), and would have the advantage of being then subject to less danger of mortality owing to their greater age. Thus the fatality rate in St. Pancras was shown to fall from 7 at age 1 year to 1.2 at 3 years, and from 2.9 at 2 years to 0.6 at 4 years of age. If the immunization were carried out on the seventh day after the rash appeared in the primary case instead of earlier, these 117 children would have attenuated attacks and become permanently immune, a much more satisfactory result under ordinary circumstances. We may roughly estimate how many home contacts would have to be immunized by serum in order to produce this salutary result on 117 children as follows. From St. Pancras data of the 1926 epidemic 80 per cent. of children under 7 who had not previously had measles, and who were living in houses where at least one case occurred, were attacked, including both the primary and the secondary cases. In an epidemic totalling 1,000 cases, from Table II, $91 \times 1.5$ or 136, would be secondary cases after ten to eighteen days' interval, and 864 would be first cases in houses. The number of children under 7 at risk before the epidemic, assuming the St. Pancras figures to apply to Battersea, would be $950 / .8=1,188$, and deducting those of the 864 primary cases who were under 7 , estimated at 821 , there would be 367 home contacts under 7 years of age who had not had measles, of whom 129 would be expected to contract the disease immediately. In our district producing an epidemic of 1,000 cases the number of home contacts to be immunized after the point $\mathrm{A}$ in Fig. 2 would be therefore about 350, and the total result of this work would be either to delay or to attenuate the attack in one-third of them.

\section{Effect of Immunizing All 5-year-old Children}

Let us now suppose that at point $A$ on the curve in Fig. 2, that is when the incidence in ten days has exceeded 5 per cent. of the total size of the epidemic expected from previous experience, all the 5-year-old children who have not had measles are immunized. It is known that the passive immunity produced falls to a level which is ineffective in stopping an attack after two to four weeksthat is to say, in some children it will have fallen to this level in two weeks and in others not until four weeks have elapsed. The rate. of fall of artificially produced immunity to various organisms has been experimentally studied in a few instances, and it seems reasonable to infer that the fall in passive immunity begins at once, and takes place at a uniform rate.

Herrman $^{5}$ found that after the second month of age the percentages of children (of mothers who had at some time had measles) who exhibited immunity to measles infection were in successive months of age as follows: $95,90,75,55,34,12,5$. These figures indicate, it is believed, the loss of passive immunity derived in the first instance by the infant through the placental circulation, and it is to be expected that passive immunity artificially produced by serum injections will be lost in a similar manner-that is to say, by almost equal decrements in equal units of time, as in the diagram (Fig. 3).

In this diagram the amount of passive immunity is represented on a vertical scale, being zero at $o$, sufficient at $B$ to render an attack mild, and at $c$ just sufficient to suppress it altogether. Under normal conditions there can be few, if any, children with sufficient inherent passive immunity to suppress an attack of measles completely, but since many mild attacks occur, it is no doubt present in some children to an extent exceeding $O B$, though not to an extent approaching oc, as represented in the lefthand side of the diagram. Injection of convalescent serum at point $\mathrm{A}$ must raise the level in all the children well above the c level, but not to the same height in all, and it may be assumed that the subsequent behaviour of

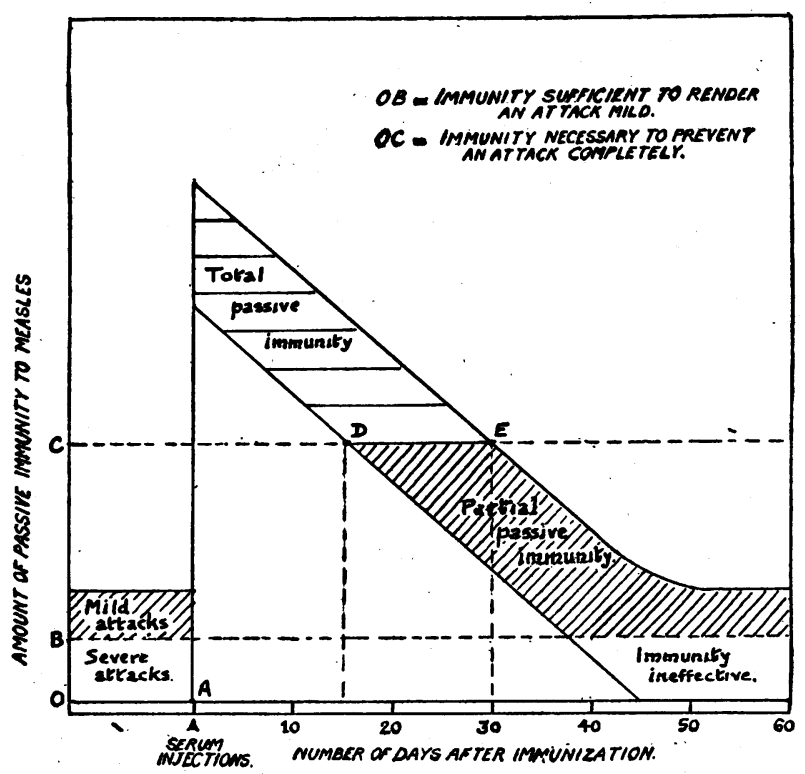

FIG. 3.-Diagrammatic representation of the probable effect of treating a group of children with measles convalescent serum.

a large group of children simultaneously immunized at A will be represented by the shaded band. During the first fifteen days all will be totally immune to infection; between fifteen and thirty days an increasing number will lose their total immunity and have only a partial immunity leading to attenuated or mild attacks if infected during this period ; from thirty. to about forty days all of them will have this partial immunity, but after about fifty days they will have returned to the original distribution.

The effect of immunizing the 5-year-olds at $A$ on the epidemic curve of Fig. 2 can now be estimated, and is shown in Table II and by the dotted line in Fig. 2. The assumptions made for simplicity are that the incubation period is ten to fifteen days; that infections received more than nine days before-serum treatment at $A$ would be unaffected by it; that infections received six to nine days before $A$ would result in mild attacks, and those received in the period from five days before $\mathrm{A}$ to fifteen days after A would all be suppressed; that infections received at any point after fifteen days would be suppressed or attenuated in the proportions indicated on a vertical line erected in Fig. 3 at that point of time. Thus infections received between fifteen and twenty days on the time scale would result in complete suppression in about 80 per cent., and attenuation in 20 per cent., and these deductions must be made from the cases which would have arisen in the undisturbed epidemic ten to fifteen days later-nañely, between twenty-five and thirty-five days after serum treatment. Proceeding 
in this way, the numbers of 5-year-olds saved from measles attack would, as shown in Table II, amount to 78 out of a total of 221 . In addition, about 35 would have mild attacks instead of severe attacks.

Assuming that all the " primary" cases really arise by infection from some other primary case ten to fifteen days before, it is an easy matter to calculate the number of children who would subsequently be saved from measles by the suppression of the 78 -year-old sources of infection, by multiplying the number suppressed in a given period by the ratio of new cases arising in a period ten to fifteen days later to those arising in the period in question according to the epidemic curve. Thus the 7 cases suppressed ten to twenty days after the point $\mathrm{A}$ would have been expected to give rise to $135 / 96$ times 7 fresh cases developing in the period twenty-five to thirty-five days after A, or 5 cases in the period twenty to thirty and 5 in the period thirty to forty. These 10 cases would again be expected to have given rise to others further on, which can be calculated in the same way. Thus the chain of 215 children who would be indirectly saved from measles attack is arrived at, giving a total of 293 cases suppressed out of 1,000. The modification of the epidemic curve thus produced is shown by the dotted line in Fig. 2.

In this calculation it is of course assumed that the children thus saved from .measles would not contract infections from other sources than those which have been removed, and also takes no account of the fact that the epidemic might by such interference be merely prolonged until almost the full 1,000 children had been attacked. This last event is not an unlikely one if the theory of the natural termination of epidemics of measles through temporary latent active immunization of the bulk of the child population is correct. Nevertheless, the calculation serves to show the utmost possible effect which might be produced through treating all the 5-yearolds who had not had measles with serum in the early stages of an epidemic. The number who would have to be so treated may be estimated thus. From St. Pancras data $^{4}$ it appears that the number of 5-year-old children at risk at the start of an epidemic is approximately equal to the total children of all ages who are subsequently attacked in the epidemic, and on this basis there would be about 900 requiring immunization at point $\mathrm{A}$, the utmost result of this being to delay the attack of measles, by two years perhaps, in about 300 children.

\section{Conclusions}

The immunization of all home contacts with convalescent or parent's serum is therefore the more practical procedure, and the more certain in its results, but the total volume of an epidemic could not be reduced by more than about 13 per cent. by this means, and only about one-third of the injections would have any useful effect at all.

The second method, the immunization of all children of certain ages who have not had measles, is more problematical in its results, but presents the theoretical possibility at least of reducing an epidemic temporarily to any proportions desired, though probably only thus making inevitable a larger epidemic at a later date.

There remains another possible method of procedure which may provide the solution-namely, artificially to expose children of certain ages to measles infection, and also give them a partial immunity. Herrman advocated and carried out the inoculation of infants before they had lost their maternally derived immunity by applying the nasal discharge from a measles case to the nasal mucous membrane of children in the fifth month of age. This has the disadvantage that they may infect their older brothers and sisters in the mild attack which follows, though if adopted as a routine practice this would soon cease to occur, since they would all have been previously immunized. A better method, suggested I think by Zingher, is to apply the virus to the nasal mucous membrane of rather older children, and several days later inject convalescent serum subcutaneously so as to ensure a mild attack and permanent active immunization.

Such inoculation from child to child is of course open to serious objections, but more natural though less certain methods of exposure could be substituted. For instance, we have heard of " measles tea parties" and frowned upon them, but when coupled with subsequent artificial immunization they would cease to be barbarous, and become humane. Perhaps 50 per cent. would respond to such exposure in the way desired; the remainder would escape infection for a year or two by the combined protection of active latent immunity and artificially produced passive immunity, and would have to be either left to their own devices, or given another chance some two years later. There is no theoretical reason why by applying some variety of this method systematically measles epidemics could not be completely controlled or done away with as we know them, and the mortality and harmful sequels reduced to a negligible quantity.

Towards the expenses of research on the Battersea records, of which use has been made in this paper, a grant was made by the British Medical Association.

${ }^{1}$ Lancet, 1930, i, 802.

REFERENCES

${ }^{2}$ British Medical Journal, 1931, ii, 602.

${ }^{3}$ Lancet, 1930, i, 801 (Fig. 5).

Annals of Eugenics, 1928, iii, 3-4, 361.

${ }^{5}$ Herrman, C.: New York State Journ. Med., October, 1923.

${ }^{6}$ British Medical Journal, 1931, i, 157.

\section{A NOTE ON SEPTICAEMIA*}

BY

SIR W. I. DE C. WHEELER

The largest contribution which surgery has made towards the treatment of septicaemia is in the field of prophylaxis. A discussion on prophylaxis would revolve naturally round Listerism, but it is too wide a sphere for a meeting such as this.

A few weak links in the prophylactic chain are worthy of consideration. We know, for example, that if there is a discoverable focus of infection which can be adequately dealt with, the infected blood stream usually takes care of itself and the prognosis becomes good. The search for this microbic fountain may tax all our resources; it may be found in the gall-bladder or in the root of a tooth. The onset of septicaemia is often traceable to the injudicious management of a primary focusfor example, early incision or milking of an abscess near the lips is attended by real danger. I have known of four deaths from such interference. I have also seen a fatal case of acute septicaemia following injury by a razor to a septic pimple on the face. Rowlands mentioned a case of septicaemia following the too free incision of a furuncle in the external ear. By too early incision of a facial focus a rich plexus of veins may be wounded; the organisms enter the opened venous radicles and are carried via the facial. vein to the cavernous sinus, with fatal results. Again, death from general sepsis may follow prostatectomy in cases which have been - treater beforehand by radiation. It seems-probable that the epithelium of the rectum occasionally is so injured by

* Introductory remarks in a joint discussion in the Sections of Medicine and Surgery at the Annual Meeting of the British Medical Association, Eastbourne, 1931. 